R. Takahashi

Nagoya Math. J.

Vol. 189 (2008), 1-25

\title{
DIRECT SUMMANDS OF SYZYGY MODULES OF THE RESIDUE CLASS FIELD
}

\author{
RYO TAKAHASHI
}

\section{Dedicated to Professor Shiro Goto on the occasion of his sixtieth birthday}

\begin{abstract}
Let $R$ be a commutative Noetherian local ring. This paper deals with the problem asking whether $R$ is Gorenstein if the $n$th syzygy module of the residue class field of $R$ has a non-trivial direct summand of finite Gdimension for some $n$. It is proved that if $n$ is at most two then it is true, and moreover, the structure of the ring $R$ is determined essentially uniquely.
\end{abstract}

\section{$\S 1$. Introduction}

Throughout the present paper, we assume that all rings are commutative Noetherian local rings and all modules are finitely generated modules.

G-dimension is a homological invariant of a module which has been introduced by Auslander [1]. This invariant is an analogue of projective dimension. Whereas the finiteness of projective dimension characterizes the regular property of the base ring, the finiteness of G-dimension characterizes the Gorenstein property of the base ring. To be precise, any module over a Gorenstein local ring has finite G-dimension, and a local ring with residue class field of finite G-dimension is Gorenstein. G-dimension shares a lot of properties with projective dimension. For example, it also satisfies an Auslander-Buchsbaum-type equality, which is called the Auslander-Bridger formula.

Dutta [9] proved the following theorem in his research into the homological conjectures:

Theorem 1.0.1. (Dutta) Let $(R, \mathfrak{m}, k)$ be a local ring. Suppose that the $n$th syzygy module of $k$ has a non-zero direct summand of finite projective dimension for some $n \geq 0$. Then $R$ is regular.

Received July 22, 2005.

Revised December 18, 2006.

2000 Mathematics Subject Classification: Primary 13D02; Secondary 13D05, 13 H10. 
Since G-dimension is similar to projective dimension, this theorem naturally leads us to the following question:

Question 1.0.2. Let $(R, \mathfrak{m}, k)$ be a local ring. Suppose that the $n$th syzygy module of $k$ has a non-zero direct summand of finite G-dimension for some $n \geq 0$. Then is $R$ Gorenstein?

It is obviously seen from the indecomposability of $k$ that this question is true if $n=0$. Hence this question is worth considering just in the case where $n \geq 1$.

We are able to answer in this paper that the above question is true if $n \leq 2$. Furthermore, as the theorems below say, we can even determine the structure of a ring satisfying the assumption of the above question for $n=1,2$.

The organization of this paper is as follows. In Section 2, we will prepare some notions and results for later use. The definition and properties of Gdimension will be given in this section. In Section 3, we shall state the main theorems of this paper. Firstly, we will consider a local ring such that the first syzygy module of the residue class field, namely, the maximal ideal, is decomposable. We will obtain the following result:

TheOREm A. Let $(R, \mathfrak{m})$ be a complete local ring. The following conditions are equivalent:

(1) There is an R-module $M$ with $\mathrm{G}-\operatorname{dim}_{R} M<\infty=\operatorname{pd}_{R} M$, and $\mathfrak{m}$ is decomposable;

(2) $R$ is Gorenstein, and $\mathfrak{m}$ is decomposable;

(3) There are a complete regular local ring $S$ of dimension two and a regular system of parameters $x, y$ of $S$ such that $R \cong S /(x y)$.

Secondly, we will investigate a local ring such that the second syzygy module of the residue class field is decomposable, and obtain the following result:

Theorem B. Let $(R, \mathfrak{m}, k)$ be a complete local ring. (Denote by $\Omega_{R}^{2} k$ the second syzygy module of $k$.) Suppose that $\mathfrak{m}$ is indecomposable. Then the following conditions are equivalent:

(1) There is a non-trivial direct summand $M$ of $\Omega_{R}^{2} k$ with $\mathrm{G}-\operatorname{dim}_{R} M<$ $\infty$; 
(2) $R$ is Gorenstein, and $\Omega_{R}^{2} k$ is decomposable;

(3) There are a complete regular local ring $(S, \mathfrak{n})$ of dimension three, a regular system of parameters $x, y, z$ of $S$, and $f \in \mathfrak{n}$ such that $R \cong$ $S /(x y-z f)$.

Theorems A and B especially say that a complete Gorenstein local ring such that the first or second syzygy module of the residue class field is decomposable is a hypersurface, and moreover, its ring structure can be determined concretely. We will actually prove in Section 3 more general results than the above two theorems.

\section{§2. Preliminaries}

Throughout this section, let $(R, \mathfrak{m}, k)$ be a local ring. In this section, we will recall several basic notions and state related results to explain and prove the main theorems of this paper.

\section{1. (Pre)covers and (pre)envelopes}

We begin by recalling the notions of a (pre)cover and a (pre)envelope of a module. Let $\bmod R$ denote the category of finitely generated $R$-modules.

Definition 2.1.1. Let $\mathcal{C}$ be a full subcategory of $\bmod R$.

(1) Let $\phi: X \rightarrow M$ be a homomorphism from $X \in \mathcal{C}$ to $M \in \bmod R$.

(i) We call $\phi$ or $X$ a $\mathcal{C}$-precover of $M$ if for any homomorphism $\phi^{\prime}$ : $X^{\prime} \rightarrow M$ with $X^{\prime} \in \mathcal{C}$ there exists a homomorphism $f: X^{\prime} \rightarrow X$ such that $\phi^{\prime}=\phi f$.

(ii) Assume that $\phi$ is a $\mathcal{C}$-precover of $M$. We call $\phi$ or $X$ a $\mathcal{C}$-cover of $M$ if any endomorphism $f$ of $X$ with $\phi=\phi f$ is an automorphism.

(2) Let $\phi: M \rightarrow X$ be a homomorphism from $M \in \bmod R$ to $X \in \mathcal{C}$.

(i) We call $\phi$ or $X$ a $\mathcal{C}$-preenvelope of $M$ if for any homomorphism $\phi^{\prime}: M \rightarrow X^{\prime}$ with $X^{\prime} \in \mathcal{C}$ there exists a homomorphism $f: X \rightarrow$ $X^{\prime}$ such that $\phi^{\prime}=f \phi$.

(ii) Assume that $\phi$ is a $\mathcal{C}$-preenvelope of $M$. We call $\phi$ or $X$ a $\mathcal{C}$ envelope of $M$ if any endomorphism $f$ of $X$ with $\phi=f \phi$ is an automorphism.

A $\mathcal{C}$-precover (resp. $\mathcal{C}$-cover, $\mathcal{C}$-preenvelope, $\mathcal{C}$-envelope) is also called a right $\mathcal{C}$-approximation (resp. right minimal $\mathcal{C}$-approximation, left $\mathcal{C}$-approximation, left minimal $\mathcal{C}$-approximation). A $\mathcal{C}$-cover (resp. $\mathcal{C}$-envelope) is 
uniquely determined up to isomorphism whenever it exists. In general, it is uncertain whether the existence of a $\mathcal{C}$-precover (resp. $\mathcal{C}$-preenvelope) implies the existence of a $\mathcal{C}$-cover (resp. $\mathcal{C}$-envelope). However, it is true under a few assumptions: if $R$ is Henselian and $\mathcal{C}$ is closed under direct summands, then for a given $\mathcal{C}$-precover (resp. $\mathcal{C}$-preenvelope), we can extract a $\mathcal{C}$-cover (resp. $\mathcal{C}$-envelope) from it, as follows.

Proposition 2.1.2. Let $\mathcal{C}$ be a full subcategory of $\bmod R$ which is closed under direct summands. Suppose that $R$ is Henselian.

(1) Let $0 \rightarrow N \rightarrow X \stackrel{\phi}{\rightarrow} M$ be an exact sequence of $R$-modules where $\phi$ is a $\mathcal{C}$-precover of $M$. Then there exists a commutative diagram

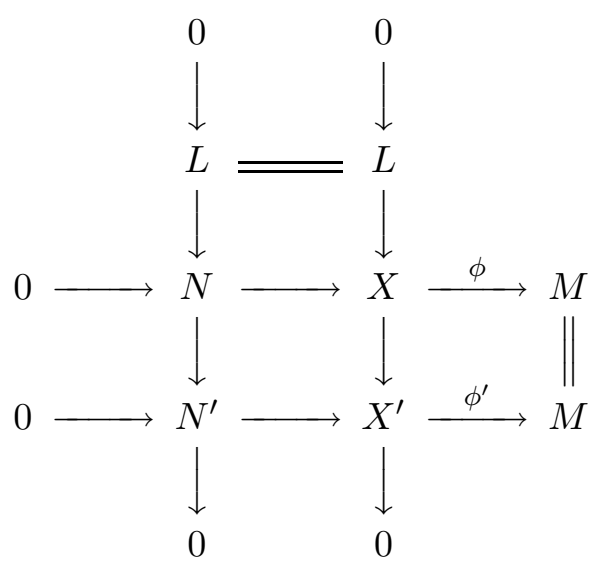

of $R$-modules with exact rows and split exact columns such that $\phi^{\prime}$ is a $\mathcal{C}$-cover of $M$.

(2) Let $M \stackrel{\phi}{\rightarrow} X \rightarrow N \rightarrow 0$ be an exact sequence of $R$-modules where $\phi$ is a $\mathcal{C}$-preenvelope of $N$. Then there exists a commutative diagram

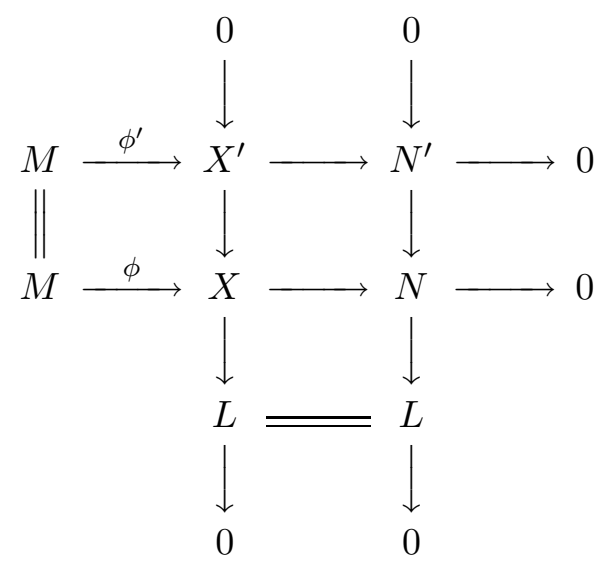


of $R$-modules with exact rows and split exact columns such that $\phi^{\prime}$ is a $\mathcal{C}$-envelope of $M$.

For the proof of the statement (1), we refer to [13, Remark 2.6]. The statement (2) can be shown dually.

\subsection{The subcategory of free modules}

We denote by $\mathcal{F}(R)$ the full subcategory of $\bmod R$ consisting of all free $R$-modules. Recall that a homomorphism $f: M \rightarrow N$ of $R$-modules is said to be minimal if the induced homomorphism $f \otimes_{R} k: M \otimes_{R} k \rightarrow N \otimes_{R} k$ is an isomorphism. (Note from Nakayama's lemma that every minimal homomorphism is surjective.) Let $\nu_{R}(M)$ denote the minimal number of generators of an $R$-module $M$, i.e., $\nu_{R}(M)=\operatorname{dim}_{k}\left(M \otimes_{R} k\right)$. Set $(-)^{*}=\operatorname{Hom}_{R}(-, R)$. Every $R$-module admits an $\mathcal{F}(R)$-cover and an $\mathcal{F}(R)$-envelope, as follows.

Proposition 2.2.1. Let $M$ be an $R$-module.

(1) A homomorphism $\phi: R^{n} \rightarrow M$ is an $\mathcal{F}(R)$-cover of $M$ if and only if $\phi$ is surjective and $n=\nu_{R}(M)$.

(2) Let $f_{1}, f_{2}, \ldots, f_{n}$ be a minimal system of generators of $M^{*}$. Then the homomorphism $f={ }^{t}\left(f_{1}, \ldots, f_{n}\right): M \rightarrow R^{n}$ is an $\mathcal{F}(R)$-envelope of $M$.

An $R$-module $M$ is said to be torsionless (resp. reflexive) if the natural homomorphism $M \rightarrow M^{* *}$ is injective (resp. bijective). We easily obtain the following.

COROllary 2.2.2. Let $M$ be an R-module.

(1) Let $\sigma: M \rightarrow M^{* *}$ be the natural homomorphism and $\phi: F \rightarrow M^{*}$ an $\mathcal{F}(R)$-cover. Then the composite map $\phi^{*} \sigma: M \rightarrow F^{*}$ is an $\mathcal{F}(R)$ envelope.

(2) The $R$-module $M$ is torsionless if and only if the $\mathcal{F}(R)$-envelope of $M$ is an injective homomorphism.

We especially see from this corollary that an $\mathcal{F}(R)$-envelope is not necessarily an injective homomorphism.

Let $M$ be an $R$-module. Take its $\mathcal{F}(R)$-cover $\pi: F \rightarrow M$. The first syzygy module $\Omega_{R} M=\Omega_{R}^{1} M$ of $M$ is defined to be the kernel of the homomorphism $\pi$, and the nth syzygy module $\Omega_{R}^{n} M$ of $M$ is defined inductively: $\Omega_{R}^{n} M=\Omega_{R}\left(\Omega_{R}^{n-1} M\right)$ for $n \geq 2$. Dually to this, we can define the cosyzygy modules of any module. 
Definition 2.2.3. Let $M$ be an $R$-module.

(1) Take the $\mathcal{F}(R)$-envelope $\theta: M \rightarrow F$ of $M$. We set $\Omega_{R}^{-1} M=\operatorname{Coker} \theta$, and call it the first cosyzygy module of $M$.

(2) Let $n \geq 2$. Assume that the $(n-1)$ th cosyzygy module $\Omega_{R}^{-(n-1)} M$ is defined. Then we set $\Omega_{R}^{-n} M=\Omega_{R}^{-1}\left(\Omega_{R}^{-(n-1)} M\right)$ and call it the $n$th cosyzygy module of $M$.

A module is said to be stable if it has no non-zero free summand. The following is a property which is peculiar to cosyzygy modules.

Proposition 2.2.4. For any $M \in \bmod R$ and any $n \geq 1$, the module $\Omega_{R}^{-n} M$ is stable.

\subsection{G-dimension}

Now, we recall the definition of G-dimension.

Definition 2.3.1. (1) We denote by $\mathcal{G}(R)$ the full subcategory of $\bmod R$ consisting of all $R$-modules $M$ satisfying the following three conditions:

(i) $M$ is reflexive,

(ii) $\operatorname{Ext}_{R}^{i}(M, R)=0$ for every $i>0$,

(iii) $\operatorname{Ext}_{R}^{i}\left(M^{*}, R\right)=0$ for every $i>0$.

(2) Let $M$ be an $R$-module. If $n$ is a non-negative integer such that there is an exact sequence $0 \rightarrow G_{n} \rightarrow G_{n-1} \rightarrow \cdots \rightarrow G_{1} \rightarrow G_{0} \rightarrow M \rightarrow 0$ of $R$-modules with $G_{i} \in \mathcal{G}(R)$ for every $i$, then we say that $M$ has $G$-dimension at most $n$, and write $\mathrm{G}-\operatorname{dim}_{R} M \leq n$. If such an integer $n$ does not exist, then we say that $M$ has infinite $G$-dimension, and write G-dim $R=\infty$.

If an $R$-module $M$ has $\mathrm{G}$-dimension at most $n$ but does not have $\mathrm{G}$ dimension at most $n-1$, then we say that $M$ has $G$-dimension $n$, and write G- $\operatorname{dim}_{R} M=n$. Note that for an $R$-module $M$ we have $\mathrm{G}-\operatorname{dim}_{R} M=0$ if and only if $M \in \mathcal{G}(R)$, and that all free $R$-modules belong to $\mathcal{G}(R)$. For basic properties of G-dimension, we should refer to [3, Chapter 3, 4], [8, Chapter 1] and [6, Section 8]. We write down some properties of the category $\mathcal{G}(R)$. 
Proposition 2.3.2. (1) If $R$ is a Gorenstein local ring, then the category $\mathcal{G}(R)$ coincides with the full subcategory of $\bmod R$ consisting of all maximal Cohen-Macaulay modules.

(2) There exists a non-free $R$-module in $\mathcal{G}(R)$ if and only if there exists an $R$-module of finite $G$-dimension and infinite projective dimension.

(3) The following statements hold:

(i) If an $R$-module $M$ belongs to $\mathcal{G}(R)$, then so do $M^{*}, \Omega M, \Omega^{-1} M$;

(ii) Let $M, N$ be R-modules. Then $M, N$ belong to $\mathcal{G}(R)$ if and only if so does $M \oplus N$

(iii) Let $0 \rightarrow L \rightarrow M \rightarrow N \rightarrow 0$ be an exact sequence of $R$-modules. If $L, N$ belong to $\mathcal{G}(R)$, then so does $M$;

(iv) If an $R$-module $M$ belongs to $\mathcal{G}(R)$, the $R /(x)$-module $M / x M$ belongs to $\mathcal{G}(R /(x))$ for any element $x \in \mathfrak{m}$ which is $R$ - and $M$-regular.

If $R$ is Gorenstein and non-regular, then the latter condition in (2) of the above proposition holds. In fact, the $R$-module $k$ has finite G-dimension and infinite projective dimension.

We denote by $\underline{\mathcal{G}}(R)$ the full subcategory of $\mathcal{G}(R)$ consisting of all stable modules in $\mathcal{G}(R)$. The dual functor $(-)^{*}$ and the syzygy functor $\Omega(-)$ make good correspondences between the category $\underline{\mathcal{G}}(R)$ and itself.

Proposition 2.3.3. $\quad$ (1) We have an anti-equivalence of categories

$$
\underline{\mathcal{G}}(R) \longrightarrow \underline{\mathcal{G}}(R), \quad M \longmapsto M^{*}
$$

with the functor being its own quasi-inverse.

(2) We have an equivalence of categories

$$
\underline{\mathcal{G}}(R) \longrightarrow \underline{\mathcal{G}}(R), \quad M \longmapsto \Omega M
$$

having as quasi-inverse functor the functor $\underline{\mathcal{G}}(R) \rightarrow \underline{\mathcal{G}}(R), M \mapsto$ $\Omega^{-1} M$.

This proposition yields the following corollary.

Corollary 2.3.4. For an $R$-module $M$, the following are equivalent:

(1) $M$ is a non-free indecomposable module in $\mathcal{G}(R)$;

(2) $M^{*}$ is a non-free indecomposable module in $\mathcal{G}(R)$;

(3) $\Omega M$ is a non-free indecomposable module in $\mathcal{G}(R)$;

(4) $\Omega^{-1} M$ is a non-free indecomposable module in $\mathcal{G}(R)$. 


\subsection{The fundamental module}

Here we introduce the concept of the fundamental module.

Definition 2.4.1. Let $(R, \mathfrak{m}, k)$ be a Cohen-Macaulay local ring of dimension two with canonical module $K$. Then since $\operatorname{Ext}_{R}^{1}(\mathfrak{m}, K) \cong$ $\operatorname{Ext}_{R}^{2}(k, K) \cong k$, there exists a non-split exact sequence $\sigma: 0 \rightarrow K \rightarrow$ $E \rightarrow \mathfrak{m} \rightarrow 0$ which is unique up to equivalence. This sequence $\sigma$ is called the fundamental sequence of $R$ and the intermediate module $E$ is called the fundamental module of $R$.

We recall a numerical invariant of a module, which was invented by Auslander.

Definition 2.4.2. Let $R$ be a Gorenstein local ring. For an $R$-module $M$, we denote by $\delta_{R}(M)$ the maximal rank of free summands of the $\mathcal{G}(R)$ cover of $M$, and set $\delta_{R}^{i}(M)=\delta_{R}\left(\Omega_{R}^{i} M\right)$, which is called the $i$ th Auslander's $\delta$-invariant of $M$.

Lemma 2.4.3. (Auslander) Let $R$ be a Gorenstein non-regular local ring with residue class field $k$. Then $\delta_{R}^{i}(k)=0$ for every $i \geq 0$. In other words, every syzygy module of $k$ admits a stable $\mathcal{G}(R)$-cover.

This lemma was proved by Auslander in the unpublished paper [2]. For the proof, we can refer to [11, Theorem 6], [4, Proposition 5.7], or [16, Theorem (4.8)].

Now, we can investigate several properties of the fundamental module of a Gorenstein local ring of dimension two.

Proposition 2.4.4. Let $R$ be a Henselian Gorenstein non-regular local ring of dimension two, and let $\sigma: 0 \rightarrow R \rightarrow E \stackrel{\phi}{\rightarrow} \mathfrak{m} \rightarrow 0$ be the fundamental sequence of $R$. Then

(1) $\phi$ is the $\mathcal{G}(R)$-cover of $\mathfrak{m}$,

(2) $E$ is stable,

(3) $E \cong \Omega_{R}^{-1}\left(\Omega_{R}^{2} k\right)$,

(4) $E$ is indecomposable if and only if so is $\Omega_{R}^{2} k$.

Proof. (1) Since $R$ is Gorenstein, $\mathcal{G}(R)$ coincides with the category of maximal Cohen-Macaulay $R$-modules, and the assertion is a well-known fact on the fundamental sequence. 
(2) This assertion follows from (1) and Lemma 2.4.3.

(3) Set $M=\Omega_{R}^{2} k$. Note that the module $M$ belongs to $\mathcal{G}(R)$. There is also an exact sequence $0 \rightarrow M \stackrel{\alpha}{\rightarrow} R^{e} \rightarrow \mathfrak{m} \rightarrow 0$. Take a minimal homomorphism $\beta: R^{r} \rightarrow M^{*}$. Then $\operatorname{Coker}\left(\beta^{*}\right)$ is isomorphic to $\Omega_{R}^{-1} M$ by definition. The dual homomorphism $\alpha^{*}:\left(R^{e}\right)^{*} \rightarrow M^{*}$ factors through $\beta$, i.e., there exists a homomorphism $\gamma:\left(R^{e}\right)^{*} \rightarrow R^{r}$ such that $\alpha^{*}=\beta \gamma$. Hence we have $\alpha=\alpha^{* *}=\gamma^{*} \beta^{*}$. Since $R \cong R^{*}$ and $M \cong M^{* *}$, we see that there is a commutative diagram

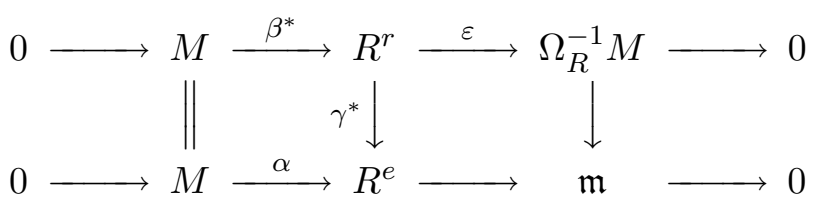

with exact rows. Take a minimal homomorphism $\zeta: R^{s} \rightarrow \operatorname{Coker}\left(\gamma^{*}\right)$ and let $\eta: R^{e} \rightarrow \operatorname{Coker}\left(\gamma^{*}\right)$ be the natural surjection. Then there is a homomorphism $\theta: R^{s} \rightarrow R^{e}$ such that $\zeta=\eta \theta$. We easily see that the homomorphism $\left(\gamma^{*}, \theta\right): R^{r} \oplus R^{s} \rightarrow R^{e}$ is surjective, and obtain a commutative diagram

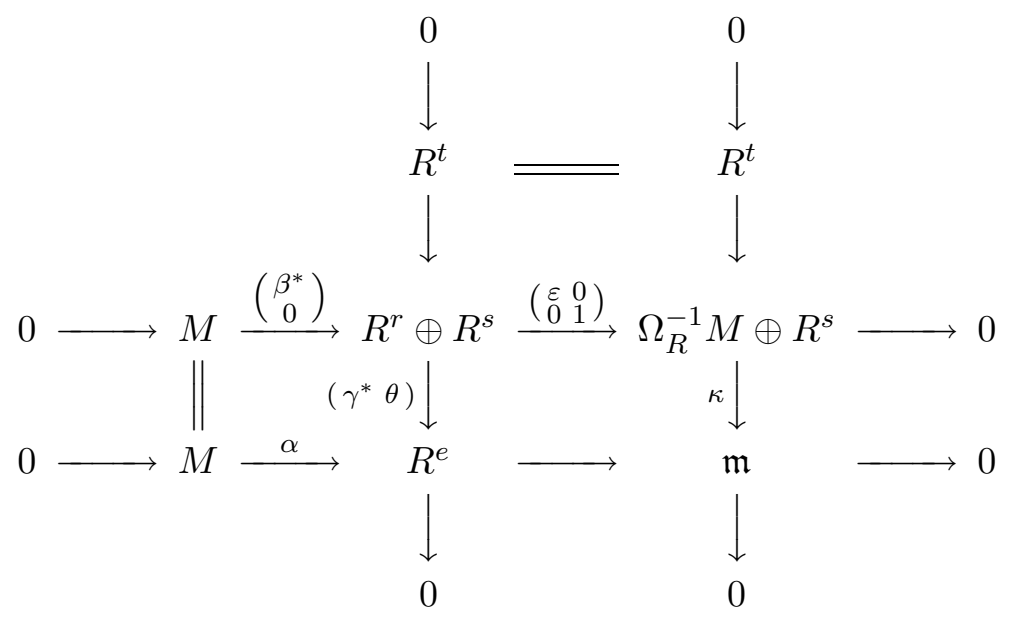

with exact rows and columns, where $t=r+s-e$. Hence the homomorphism $\kappa$ is a $\mathcal{G}(R)$-precover of $\mathfrak{m}$. It follows from (1) that there is an isomorphism $\Omega_{R}^{-1} M \oplus R^{s} \cong E \oplus R^{t-1}$. Since both $E$ and $\Omega_{R}^{-1} M$ are stable by (2), we conclude from the Krull-Schmidt theorem that the module $E$ is isomorphic to $\Omega_{R}^{-1} M$, as desired.

(4) This assertion is proved by (3). 


\section{§3. Main results}

In this section, using the results given in the previous section, we shall state and prove our main theorems.

\subsection{Idealizations}

First of all, we consider an idealization possessing a non-free reflexive module. We begin with making an easy lemma, which will often be used later.

LEMma 3.1.1. Let $(R, \mathfrak{m})$ be a local ring, $\theta: \mathfrak{m} \rightarrow R$ the natural inclusion map, and $M$ a stable $R$-module. Then the induced injective homomorphism

$$
\operatorname{Hom}_{R}(M, \theta): \operatorname{Hom}_{R}(M, \mathfrak{m}) \longrightarrow M^{*}
$$

is an isomorphism.

Proof. If there is a homomorphism from $M$ to $R$ which does not factor through $\theta$, then it is a surjection, hence is a split-epimorphism, contrary to the stability of $M$.

Now we can prove the following result.

Proposition 3.1.2. Let $(S, \mathfrak{n}, k)$ be a local ring, $V \neq 0$ a finitedimensional k-vector space, and $R=S \ltimes V$ the idealization of $V$ over $S$. Let $M$ be a non-free indecomposable reflexive $R$-module. Then

(1) $M \cong \operatorname{Soc} R \cong V \cong k$,

(2) If $\operatorname{depth} S=0$, then $S=k$, hence $R \cong k[[X]] /\left(X^{2}\right)$.

Proof. (1) Denote by $\mathfrak{m}$ the unique maximal ideal of $R$, and set $I=$ $\mathfrak{n} \ltimes 0=\{(s, v) \in R \mid s \in \mathfrak{n}, v=0\}$, and $J=0 \ltimes V=\{(s, v) \in R \mid s=0\}$. These are ideals of $R$, and it is easy to see that $\mathfrak{m}=I \oplus J$. By virtue of Lemma 3.1.1, we have isomorphisms $M^{*} \cong \operatorname{Hom}_{R}(M, \mathfrak{m}) \cong \operatorname{Hom}_{R}(M, I \oplus$ $J) \cong \operatorname{Hom}_{R}(M, I) \oplus \operatorname{Hom}_{R}(M, J)$. Since $M^{*}$ is also indecomposable, we have either $\operatorname{Hom}_{R}(M, I)=0$ or $\operatorname{Hom}_{R}(M, J)=0$. However $J$ is isomorphic to $k^{e}$ as an $R$-module where $e=\operatorname{dim}_{k} V$, hence $\operatorname{Hom}_{R}(M, J) \cong k^{n e} \neq 0$ where $n=\nu_{R}(M)$. It follows that

$$
\operatorname{Hom}_{R}(M, I)=0
$$

and $M^{*} \cong k^{n e}$. The indecomposability of $M^{*}$ again implies that $M^{*} \cong k$ and $n e=1$, hence $e=1$. Therefore $V \cong k$. Also, we have isomorphisms 
$M \cong M^{* *} \cong k^{*} \cong k^{r}$ where $r=\operatorname{dim}_{k}(\operatorname{Soc} R)$. The indecomposability of $M$ implies that $M \cong k$ and $r=1$. Hence $\operatorname{Soc} R \cong k$.

(2) Note from (3.1.2.1) and (1) that $\operatorname{Hom}_{R}(k, I)=0$. Suppose that $I \neq 0$. Then there exists an $I$-regular element $(s, v) \in \mathfrak{m}$ (cf. [7, Proposition 1.2.3]). It is easy to observe that the element $s \in \mathfrak{n}$ is $S$-regular, contrary to the assumption that depth $S=0$. Therefore we have $I=0$, equivalently, $S=k$. By (1) again, we obtain isomorphisms $R \cong k \ltimes k \cong k[[X]] /\left(X^{2}\right)$.

The structure of an idealization of the form in the above proposition is uniquely determined if it has at least a non-free module of G-dimension zero.

Corollary 3.1.3. Let $(S, \mathfrak{n}, k)$ be a local ring, $V \neq 0$ a finite-dimensional $k$-vector space, and $R=S \ltimes V$ the idealization of $V$ over $S$. Then the following are equivalent:

(1) There is a non-free $R$-module in $\mathcal{G}(R)$;

(2) $R$ is Gorenstein;

(3) $R \cong k[[X]] /\left(X^{2}\right)$.

Proof. $\quad(3) \Rightarrow(2)$ : This implication is obvious.

$(2) \Rightarrow(1):$ Note that $\operatorname{dim} R=\operatorname{depth} R=\min \left\{\operatorname{depth} S, \operatorname{depth}_{S} V\right\}=0$, namely, $R$ is an Artinian local ring. Hence $k$ belongs to $\mathcal{G}(R)$. Suppose that the $R$-module $k$ is free. Then $R$ is regular, and hence $R$ is a field. However, there is a non-zero element $v \in V$, and the element $(0, v) \in R$ is non-zero and nilpotent, which is a contradiction. Thus $k$ is a non-free $R$-module in $\mathcal{G}(R)$.

$(1) \Rightarrow(2)$ : Then, we see that there exists a non-free indecomposable $R$-module $M$ in $\mathcal{G}(R)$. By definition it is reflexive. Proposition 3.1.2(1) says that $M$ is isomorphic to $k$. It follows that $R$ is Gorenstein.

$(2) \Rightarrow(3)$ : Suppose that $\operatorname{depth} S>0$. Then we especially have $\operatorname{dim} R=$ $\operatorname{dim} S>0$. Since depth $R=0$, the local $\operatorname{ring} R$ is not Cohen-Macaulay, and hence $R$ is not Gorenstein, which is a contradiction. Therefore depth $S=0$, and Proposition 3.1.2(2) implies that $R \cong k[[X]] /\left(X^{2}\right)$.

\subsection{The first syzygy of the residue field (i.e. the maximal ideal)}

The decomposability of the maximal ideal and the existence of a nonfree module of G-dimension zero played essential roles in the achievement 
of Corollary 3.1.3. From now on, we consider a local ring satisfying these conditions in more general settings. First of all, let us describe the minimal free resolution of the residue class field of such a local ring.

Proposition 3.2.1. Let $(R, \mathfrak{m}, k)$ be a local ring. Suppose that there is a direct sum decomposition $\mathfrak{m}=I \oplus J$ where $I, J$ are non-zero ideals of $R$. Let $M$ be a non-free indecomposable module in $\mathcal{G}(R)$. Then there exist $x, y \in \mathfrak{m}$ such that

(1) $I=(x)$ and $J=(y)$,

(2) $(0: x)=(y)$ and $(0: y)=(x)$,

(3) $M$ is isomorphic to either $(x)$ or $(y)$.

Hence the minimal free resolution of $k$ is as follows:

$$
\cdots \stackrel{\left(\begin{array}{ll}
y & 0 \\
0 & x
\end{array}\right)}{\longrightarrow} R^{2} \stackrel{\left(\begin{array}{ll}
x & 0 \\
0 & y
\end{array}\right)}{\longrightarrow} R^{2} \stackrel{\left(\begin{array}{ll}
y & 0 \\
0 & x
\end{array}\right)}{\longrightarrow} R^{2} \stackrel{\left(\begin{array}{ll}
x & y
\end{array}\right)}{\longrightarrow} R \longrightarrow k \longrightarrow 0 .
$$

Proof. Both $M^{*}$ and $\Omega M$ are also non-free indecomposable modules in $\mathcal{G}(R)$. By virtue of Lemma 3.1.1, there are isomorphisms $M^{*} \cong$ $\operatorname{Hom}_{R}(M, \mathfrak{m})=\operatorname{Hom}_{R}(M, I \oplus J) \cong \operatorname{Hom}_{R}(M, I) \oplus \operatorname{Hom}_{R}(M, J)$. The indecomposability of $M^{*}$ implies that either $\operatorname{Hom}_{R}(M, I)=0$ or $\operatorname{Hom}_{R}(M, J)=$ 0 . We may assume that

$$
\operatorname{Hom}_{R}(M, J)=0 .
$$

There is an exact sequence

$$
0 \longrightarrow \Omega M \longrightarrow R^{n} \longrightarrow M \longrightarrow 0 \text {. }
$$

Dualizing this by $J$, we obtain another exact sequence $\operatorname{Hom}_{R}(M, J) \rightarrow$ $J^{n} \rightarrow \operatorname{Hom}_{R}(\Omega M, J)$. We have $\operatorname{Hom}_{R}(\Omega M, J) \neq 0$ by (3.2.1.1). Applying the above argument to the module $\Omega M$ yields

$$
\operatorname{Hom}_{R}(\Omega M, I)=0 .
$$

Also, dualizing (3.2.1.2) by $I$, we get an exact sequence $0 \rightarrow \operatorname{Hom}_{R}(M, I) \rightarrow$ $I^{n} \rightarrow \operatorname{Hom}_{R}(\Omega M, I)$, and hence $M^{*} \cong \operatorname{Hom}_{R}(M, I) \cong I^{n}$. The indecomposability of $M^{*}$ implies that $n=1$ (i.e. $M$ is cyclic), and $M^{*} \cong I$. Let $\alpha: M^{*} \rightarrow I$ denote this isomorphism, and write $M=R z$ for some $z \in M$. Then it is easy to check that $\alpha$ is a map defined by $\alpha(\sigma)=\sigma(z)$ for $\sigma \in M^{*}$. 
We also have $M \cong M^{* *} \cong \operatorname{Hom}_{R}\left(M^{*}, \mathfrak{m}\right) \cong \operatorname{Hom}_{R}\left(M^{*}, I\right) \oplus$ $\operatorname{Hom}_{R}\left(M^{*}, J\right)$. Note that $\operatorname{Hom}_{R}\left(M^{*}, I\right)$ is isomorphic to $\operatorname{Hom}_{R}(I, I)$, which contains the identity map of $I$. Hence $\operatorname{Hom}_{R}\left(M^{*}, I\right) \neq 0$ and therefore $\operatorname{Hom}_{R}\left(M^{*}, J\right)=0$. Applying the above argument to the module $M^{*}$, we see that $M^{*}$ is also cyclic and $M \cong M^{* *} \cong I$. Thus, we have shown that $M \cong M^{*} \cong I$ and these modules are cyclic. Noting (3.2.1.3) and applying the above argument to the module $\Omega M$, we see that $\Omega M \cong(\Omega M)^{*} \cong J$ and these modules are cyclic.

Write $I=(x)$ and $J=(y)$. Then $M$ is isomorphic to the principal ideal $(x)$. Apply the above argument to $(x)$ instead of $M$, and we have an isomorphism $\alpha:(x)^{*} \rightarrow(x)$ which is defined by $\alpha(\sigma)=\sigma(x)$ for $\sigma \in(x)^{*}$. Consider a composite map $(0:(0: x)) \stackrel{\gamma}{\rightarrow}(R /(0: x))^{*} \stackrel{\beta}{\rightarrow}(x)^{*} \stackrel{\alpha}{\rightarrow}(x)$, where $\beta, \gamma$ are natural isomorphisms. We easily see that this composite map is the identity map. Hence $(0:(0: x))=(x)$. Similarly, we also have $(0:(0: y))=(y)$. Since $(0: x)=\Omega(x) \cong \Omega M \cong(y)$, we have $(x)=(0:(0: x))=\operatorname{Ann}_{R}(0: x)=\operatorname{Ann}_{R}(y)=(0: y)$, and therefore $(0: x)=\operatorname{Ann}_{R}(x)=\operatorname{Ann}_{R}(0: y)=(0:(0: y))=(y)$. Thus we obtain the minimal free resolutions of $(x)$ and $(y)$ :

$$
\left\{\begin{array}{l}
\cdots \stackrel{y}{\longrightarrow} R \stackrel{x}{\longrightarrow} R \stackrel{y}{\longrightarrow} R \longrightarrow(x) \longrightarrow 0, \\
\cdots \stackrel{x}{\longrightarrow} R \stackrel{y}{\longrightarrow} R \stackrel{x}{\longrightarrow} R \longrightarrow(y) \longrightarrow 0 .
\end{array}\right.
$$

Taking the direct sum of these exact sequence, we get

$$
\cdots \stackrel{\left(\begin{array}{ll}
y & 0 \\
0 & x
\end{array}\right)}{\longrightarrow} R^{2} \stackrel{\left(\begin{array}{ll}
x & 0 \\
0 & y
\end{array}\right)}{\longrightarrow} R^{2} \stackrel{\left(\begin{array}{ll}
y & 0 \\
0 & x
\end{array}\right)}{\longrightarrow} R^{2} \longrightarrow \mathfrak{m} \longrightarrow 0 .
$$

Joining this to the natural exact sequence $0 \rightarrow \mathfrak{m} \rightarrow R \rightarrow k \rightarrow 0$ constructs the minimal free resolution of $k$ in the assertion.

We denote by $\operatorname{edim} R$ the embedding dimension of a local $\operatorname{ring} R$. When a homomorphic image of a regular local ring is given, we can choose a minimal presentation of the ring in the following sense:

Proposition 3.2.2. Let $R$ be a homomorphic image of a regular local ring. Then there exist a regular local ring $(S, \mathfrak{n})$ and an ideal $I$ of $S$ contained in $\mathfrak{n}^{2}$ such that $R \cong S / I$.

Here we introduce a famous result due to Tate [14, Theorem 6]. See also [5, Remarks 8.1.1(3)]. 
Lemma 3.2.3. (Tate) Let $(S, \mathfrak{n}, k)$ be a regular local ring, $I$ an ideal of $S$ contained in $\mathfrak{n}^{2}$, and $R=S / I$ a residue class ring. Suppose that the complexity of $k$ over $R$ is at most one. (In other words, the set of all the Betti numbers of the $R$-module $k$ is bounded.) Then $I$ is a principal ideal.

We denote by $\beta_{i}^{R}(M)$ the $i$ th Betti number of a module $M$ over a local ring $R$. Handling the above results, we can determine the structure of a local ring with decomposable maximal ideal having a non-free module of G-dimension zero, as follows:

THEOREM 3.2.4. Let $(S, \mathfrak{n}, k)$ be a regular local ring, $I$ an ideal of $S$ contained in $\mathfrak{n}^{2}$, and $R=S / I$ a residue class ring. Suppose that there exists a non-free $R$-module in $\mathcal{G}(R)$. Then the following conditions are equivalent:

(1) The maximal ideal of $R$ is decomposable;

(2) $\operatorname{dim} S=2$ and $I=(x y)$ for some regular system of parameter $x, y$ of $S$.

Proof. Let $\mathfrak{m}=\mathfrak{n} / I$ be the maximal ideal of $R$.

$(2) \Rightarrow(1)$ : It is easy to see that $\mathfrak{m}=x R \oplus y R$ and that $x R, y R$ are non-zero.

$(1) \Rightarrow(2)$ : First of all, note from the condition (1) that $R$ is not an integral domain, hence is not a regular local ring. Proposition 3.2.1 says that $\mathfrak{m}=x R \oplus y R$ for some $x, y \in \mathfrak{n}$, and that $\beta_{i}^{R}(k)=2$ for every $i \geq 2$. It follows from Lemma 3.2.3 that $I$ is a principal ideal. Hence $R$ is a hypersurface. We write $I=(f)$ for some $f \in \mathfrak{n}^{2}$. Since $\mathfrak{m}$ is decomposable, the local ring $R$ is not Artinian. (Over an Artinian Gorenstein local ring, the intersection of non-zero ideals is also non-zero; cf. [7, Exercise 3.2.15].) Hence we have $0<\operatorname{dim} R<\operatorname{edim} R=2$, which says that $\operatorname{dim} R=1$ and $\operatorname{dim} S=2$.

Note that $\mathfrak{n}=(x, y, f)$. Because $\operatorname{edim} S=\operatorname{dim} S=2$, one of the elements $x, y, f$ belongs to the ideal generated by the other two elements. Noting that the images of elements $x, y$ in $\mathfrak{m}$ form a minimal system of generators of $\mathfrak{m}$, we see that $f \in(x, y)$, and hence $x, y$ is a regular system of parameters of $S$. On the other hand, noting $x R \cap y R=0$, we get $x y \in I=(f)$. Write $x y=c f$ for some $c \in S$. Since the associated graded ring $\operatorname{gr}_{\mathfrak{n}}(S)$ is a polynomial ring over $k$ in two variables $\bar{x}, \bar{y} \in \mathfrak{n} / \mathfrak{n}^{2}$, we especially have $\overline{x y} \neq 0$ in $\mathfrak{n}^{2} / \mathfrak{n}^{3}$, namely, $x y \notin \mathfrak{n}^{3}$. It follows that $c \notin \mathfrak{n}$ because $f \in \mathfrak{n}^{2}$. Therefore the element $c$ is a unit of $S$, and thus $I=(x y)$. 
Using Theorem 3.2.4 and Cohen's structure theorem, we obtain the following corollary.

COROLlary 3.2.5. Let $(R, \mathfrak{m})$ be a complete local ring. The following conditions are equivalent:

(1) There is a non-free module in $\mathcal{G}(R)$, and $\mathfrak{m}$ is decomposable;

(2) $R$ is Gorenstein, and $\mathfrak{m}$ is decomposable;

(3) There are a complete regular local ring $S$ of dimension two and a regular system of parameters $x, y$ of $S$ such that $R \cong S /(x y)$.

Note that the finiteness of G-dimension is independent of completion. Thus, Corollary 3.2.5 not only gives birth to a generalization of [13, Proposition 2.3] but also guarantees that Question 1.0.2 is true if $n=1$.

\subsection{The second syzygy of the residue field}

As far as here, we have observed a local ring whose maximal ideal is decomposable. From here to the end of this paper, we will observe a local ring such that the second syzygy module of the residue class field is decomposable. We begin with the following theorem, which implies that Question 1.0.2 is true if $n=2$.

Theorem 3.3.1. Let $(R, \mathfrak{m}, k)$ be a local ring. Suppose that $\mathfrak{m}$ is indecomposable and that $\Omega_{R}^{2} k$ has a non-zero proper direct summand of finite $G$-dimension. Then $R$ is a Gorenstein ring of dimension two.

Proof. Replacing $R$ with its $\mathfrak{m}$-adic completion, we may assume that $R$ is a complete local ring. In particular, note that $R$ is Henselian. We have $\Omega_{R}^{2} k=M \oplus N$ for some non-zero $R$-modules $M$ and $N$ with G-dim ${ }_{R} M<\infty$. There is an exact sequence $0 \rightarrow M \oplus N \stackrel{(f, g)}{\longrightarrow} R^{e} \rightarrow \mathfrak{m} \rightarrow 0$ of $R$-modules, where $e=\operatorname{edim} R$. Setting $A=$ Coker $f$ and $B=$ Coker $g$, we get exact sequences

$$
\left\{\begin{array}{l}
0 \longrightarrow M \stackrel{f}{\longrightarrow} R^{e} \stackrel{\alpha}{\longrightarrow} A \longrightarrow 0, \\
0 \longrightarrow N \stackrel{g}{\longrightarrow} R^{e} \stackrel{\beta}{\longrightarrow} B \longrightarrow 0 .
\end{array}\right.
$$

It is easily observed that there are exact sequences

$$
0 \longrightarrow R^{e} \stackrel{\left(\begin{array}{l}
\alpha \\
\beta
\end{array}\right)}{\longrightarrow} A \oplus B \longrightarrow \mathfrak{m} \longrightarrow 0
$$


and

$$
\left\{\begin{array}{l}
0 \longrightarrow M \stackrel{\beta f}{\longrightarrow} B \longrightarrow \mathfrak{m} \longrightarrow 0 \\
0 \longrightarrow N \stackrel{\alpha g}{\longrightarrow} A \longrightarrow \mathfrak{m} \longrightarrow 0
\end{array}\right.
$$

Claim 1. We have $\operatorname{Ext}_{R}^{2}(k, R) \neq 0$. (Hence $\operatorname{depth} R \leq 2$.)

Proof. Suppose that $\operatorname{Ext}_{R}^{2}(k, R)=0$. Then $\operatorname{Ext}_{R}^{1}\left(\mathfrak{m}, R^{e}\right) \cong \operatorname{Ext}_{R}^{2}\left(k, R^{e}\right)$ $=0$. Hence the exact sequence (3.3.1.2) splits, and therefore we have an isomorphism $A \oplus B \cong R^{e} \oplus \mathfrak{m}$. Since the maximal ideal $\mathfrak{m}$ is indecomposable, it follows from the Krull-Schmidt theorem that $\mathfrak{m}$ is isomorphic to a direct summand of $A$ or $B$. If $\mathfrak{m}$ is isomorphic to a direct summand of $A$, then $B$ is isomorphic to a direct summand of $R^{e}$. Hence $B$ is a free $R$-module of rank at most $e$. Denote by $b$ the rank of $B$. Since the second sequence in (3.3.1.1) splits, the $R$-module $N$ is a free module of rank $e-b$. Noting that there is a surjective homomorphism from $B$ to $\mathfrak{m}$ by (3.3.1.3), we have $b=\nu_{R}(B) \geq \nu_{R}(\mathfrak{m})=e$. This means that $b=e$, and hence $N=0$, which is a contradiction. We can get a contradiction along the same lines in the case where $\mathfrak{m}$ is isomorphic to a direct summand of $B$. Thus, we obtain $\operatorname{Ext}_{R}^{2}(k, R) \neq 0$.

Fix a non-free indecomposable module $X \in \mathcal{G}(R)$. Applying the functor $\operatorname{Hom}_{R}(X,-)$ to (3.3.1.2) gives an exact sequence $0 \rightarrow\left(X^{*}\right)^{e} \rightarrow \operatorname{Hom}_{R}(X, A)$ $\oplus \operatorname{Hom}_{R}(X, B) \rightarrow \operatorname{Hom}_{R}(X, \mathfrak{m}) \rightarrow 0$ and an isomorphism

$$
\operatorname{Ext}_{R}^{1}(X, A) \oplus \operatorname{Ext}_{R}^{1}(X, B) \cong \operatorname{Ext}_{R}^{1}(X, \mathfrak{m}) .
$$

We have $\left(X^{*}\right)^{e} \in \mathcal{G}(R)$ and $\operatorname{Hom}_{R}(X, \mathfrak{m}) \in \mathcal{G}(R)$ by Lemma 3.1.1, hence $\operatorname{Hom}_{R}(X, A) \in \mathcal{G}(R)$.

Take the first syzygy module of $X$; we have an exact sequence $0 \rightarrow$ $\Omega X \rightarrow R^{n} \rightarrow X \rightarrow 0$. Dualizing this sequence by $A$, we obtain an exact sequence $0 \rightarrow \operatorname{Hom}_{R}(X, A) \rightarrow A^{n} \rightarrow \operatorname{Hom}_{R}(\Omega X, A) \rightarrow \operatorname{Ext}_{R}^{1}(X, A) \rightarrow 0$. Divide this into two short exact sequences

$$
\left\{\begin{array}{l}
0 \longrightarrow \operatorname{Hom}_{R}(X, A) \longrightarrow A^{n} \longrightarrow C \longrightarrow 0, \\
0 \longrightarrow C \longrightarrow \operatorname{Hom}_{R}(\Omega X, A) \longrightarrow \operatorname{Ext}_{R}^{1}(X, A) \longrightarrow 0
\end{array}\right.
$$

of $R$-modules. Since $\Omega X$ is also a non-free indecomposable module in $\mathcal{G}(R)$, applying the above argument to $\Omega X$ instead of $X$ shows that the module 
$\operatorname{Hom}_{R}(\Omega X, A)$ also belongs to $\mathcal{G}(R)$. We have G-dim $R\left(A^{n}\right)<\infty$ by the first sequence in (3.3.1.1). Hence it follows from (3.3.1.5) that G- $\operatorname{dim}_{R} C<\infty$, and

$$
\text { G- } \operatorname{dim}_{R}\left(\operatorname{Ext}_{R}^{1}(X, A)\right)<\infty .
$$

On the other hand, applying the functor $\operatorname{Hom}_{R}(X,-)$ to the natural exact sequence $0 \rightarrow \mathfrak{m} \rightarrow R \rightarrow k \rightarrow 0$, we get an exact sequence $0 \rightarrow$ $\operatorname{Hom}_{R}(X, \mathfrak{m}) \rightarrow X^{*} \rightarrow \operatorname{Hom}_{R}(X, k) \rightarrow \operatorname{Ext}_{R}^{1}(X, \mathfrak{m}) \rightarrow 0$. Lemma 3.1.1 implies that $\operatorname{Hom}_{R}(X, k) \cong \operatorname{Ext}_{R}^{1}(X, \mathfrak{m})$, hence $\operatorname{Ext}_{R}^{1}(X, \mathfrak{m})$ is a $k$-vector space. Since $\operatorname{Ext}_{R}^{1}(X, A)$ is contained in $\operatorname{Ext}_{R}^{1}(X, \mathfrak{m})$ by (3.3.1.4),

$$
\operatorname{Ext}_{R}^{1}(X, A) \text { is a } k \text {-vector space. }
$$

Claim 2. The local ring $R$ is Gorenstein.

Proof. Suppose that $R$ is not Gorenstein. Then we must have $\operatorname{Ext}_{R}^{1}(G, A)=0$ for any $G \in \mathcal{G}(R)$ by (3.3.1.6) and (3.3.1.7). We have an exact sequence

$$
0 \longrightarrow X \longrightarrow R^{m} \longrightarrow \Omega^{-1} X \longrightarrow 0,
$$

and note that $\Omega^{-1} X$ belongs to $\mathcal{G}(R)$. The exact sequences (3.3.1.8) and (3.3.1.1) yield isomorphisms

$$
\operatorname{Ext}_{R}^{1}(X, M) \cong \operatorname{Ext}_{R}^{2}\left(\Omega^{-1} X, M\right) \cong \operatorname{Ext}_{R}^{1}\left(\Omega^{-1} X, A\right)=0 .
$$

This means that

$$
\operatorname{Ext}_{R}^{1}(G, M)=0
$$

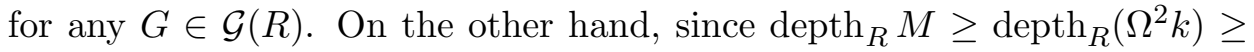
$\min \{2, \operatorname{depth} R\}=\operatorname{depth} R$ by [7, Exercise 1.3.7] and Claim 1, $M$ belongs to $\mathcal{G}(R)$. Hence there is an exact sequence of the form $0 \rightarrow M \rightarrow R^{l} \rightarrow$ $\Omega^{-1} M \rightarrow 0$, and this splits because $\operatorname{Ext}_{R}^{1}\left(\Omega^{-1} M, M\right)=0$ by (3.3.1.9). Thus the $R$-module $M$ is free. Theorem 1.0.1 implies that $R$ is regular, which contradicts our assumption that $R$ is not Gorenstein. This contradiction proves the claim.

Since the only number $i$ such that $\operatorname{Ext}_{R}^{i}(k, R) \neq 0$ is the Krull dimension of $R$ if $R$ is Gorenstein, it follows from the above two claims that $R$ is a Gorenstein local ring of dimension two, which completes the proof of the theorem. 
The above theorem interests us in the investigation of a Gorenstein local ring of dimension two such that the second syzygy module of the residue class field is decomposable. Our result concerning this is stated as follows.

TheOREm 3.3.2. Let $(S, \mathfrak{n}, k)$ be a regular local ring, $I$ an ideal of $S$ contained in $\mathfrak{n}^{2}$, and $R=S / I$ a residue class ring. Suppose that $R$ is a Henselian Gorenstein ring of dimension two. Then the following are equivalent:

(1) $\Omega_{R}^{2} k$ is decomposable;

(2) $\operatorname{dim} S=3$ and $I=(x y-z f)$ for some regular system of parameters $x, y, z$ of $S$ and $f \in \mathfrak{n}$.

It is necessary to prepare three elementary lemmas to prove this theorem. The first and third ones are both well-known and easy to check, and we omit the proofs.

Lemma 3.3.3. Let $(S, \mathfrak{n}, k)$ be a regular local ring of dimension three and $R=S /(f)$ a hypersurface with $f \in \mathfrak{n}^{2}$. Then $f=x f_{x}+y f_{y}+z f_{z}$ for some $f_{x}, f_{y}, f_{z} \in \mathfrak{n}$, and the minimal free resolution of $k$ over $R$ is as follows:

$$
\ldots \stackrel{C}{\longrightarrow} R^{4} \stackrel{D}{\longrightarrow} R^{4} \stackrel{C}{\longrightarrow} R^{4} \stackrel{D}{\longrightarrow} R^{4} \stackrel{C}{\longrightarrow} R^{4} \stackrel{B}{\longrightarrow} R^{3} \stackrel{A}{\longrightarrow} R \longrightarrow k \longrightarrow 0,
$$

where

$$
\begin{array}{rlrl}
A & =\left(\begin{array}{lll}
x & y & z
\end{array}\right), & B & =\left(\begin{array}{cccc}
0 & -z & y & f_{x} \\
z & 0 & -x & f_{y} \\
-y & x & 0 & f_{z}
\end{array}\right), \\
C=\left(\begin{array}{ccccc}
0 & -f_{z} & f_{y} & x \\
f_{z} & 0 & -f_{x} & y \\
-f_{y} & f_{x} & 0 & z \\
-x & -y & -z & 0
\end{array}\right), & D=\left(\begin{array}{cccc}
0 & -z & y & f_{x} \\
z & 0 & -x & f_{y} \\
-y & x & 0 & f_{z} \\
-f_{x} & -f_{y} & -f_{z} & 0
\end{array}\right) .
\end{array}
$$

Lemma 3.3.4. Let $(R, \mathfrak{m}, k)$ be a local ring and $x \in \mathfrak{m}-\mathfrak{m}^{2}$ an $R$-regular element. Then we have a split exact sequence $0 \rightarrow k \stackrel{\theta}{\rightarrow} \mathfrak{m} / x \mathfrak{m} \stackrel{\pi}{\rightarrow} \mathfrak{m} / x R \rightarrow 0$, where $\theta$ is defined by $\theta(\bar{a})=\overline{x a}$ for $\bar{a} \in R / \mathfrak{m}=k$ and $\pi$ is the natural surjection.

Proof. Let $x_{1}, x_{2}, \ldots, x_{n}$ be a minimal system of generators of $\mathfrak{m}$ with $x_{1}=x$. Define a homomorphism $\varepsilon: \mathfrak{m} / x \mathfrak{m} \rightarrow k$ by $\varepsilon\left(\sum_{i=1}^{n} \overline{x_{i} a_{i}}\right)=\overline{a_{1}}$. We easily see that the composite map $\varepsilon \theta$ is the identity map of $k$, which means that $\theta$ is a split-monomorphism. 
Lemma 3.3.5. Let $(R, \mathfrak{m}, k)$ be a Cohen-Macaulay local ring of dimension one. Then $R$ is a discrete valuation ring if and only if $\mathfrak{m}^{*}$ is a cyclic $R$-module.

Now let us prove Theorem 3.3.2.

Proof of Theorem 3.3.2. (2) $\Rightarrow(1)$ : We have $x y-z f=x \cdot 0+y \cdot x+z \cdot$ $(-f)$. Lemma 3.3.3 gives a finite free presentation $R^{4} \stackrel{C}{\longrightarrow} R^{4} \rightarrow \Omega_{R}^{2} k \rightarrow 0$ of the $R$-module $\Omega_{R}^{2} k$, where $C=\left(\begin{array}{cccc}0 & f & x & x \\ -f & 0 & 0 & y \\ -x & 0 & 0 & z \\ -x & -y & -z & 0\end{array}\right)$. Putting $P=\left(\begin{array}{cccc}1 & 0 & 0 & 0 \\ 0 & 0 & 1 & -1 \\ 0 & 0 & 1 & 0 \\ 0 & 1 & 0 & 0\end{array}\right)$ and $Q=\left(\begin{array}{cccc}0 & 0 & -1 & 0 \\ 0 & 1 & 0 & 0 \\ 1 & 0 & 0 & -1 \\ 0 & 0 & 0 & 1\end{array}\right)$, we obtain $P C Q=\left(\begin{array}{cc}U & 0 \\ 0 & { }^{t} U\end{array}\right)$, where $U=\left(\begin{array}{ll}x & f \\ z & y\end{array}\right)$. It is easily seen that the matrices $P, Q$ are invertible. Denoting by $M$ (resp. $N)$ the cokernel of the homomorphism defined by the matrix $U$ (resp. ${ }^{t} U$ ), we get an isomorphism $\Omega_{R}^{2} k \cong M \oplus N$.

$(1) \Rightarrow(2)$ : First of all, note that the local ring $R$ is not regular. We denote by $\mathfrak{m}$ the maximal ideal $\mathfrak{n} / I$ of $R$.

Suppose that there exists an element $z \in \mathfrak{n}-\mathfrak{n}^{2}$ whose image in $\mathfrak{m}$ is an $R$-regular element such that the module $\mathfrak{m} / z R$ is decomposable. Then the assertion (2) follows. Indeed, put $\overline{(-)}=(-) \otimes_{S} S /(z)$. Note that $\bar{S}$ is also a regular local ring because $z$ is a minimal generator of the maximal ideal $\mathfrak{n}$ of $S$ (see the proof of Proposition 3.2.2). Since the maximal ideal $\mathfrak{m} \bar{R}$ of $\bar{R}$ is decomposable, we can apply Theorem 3.2.4 and see that $\operatorname{dim} \bar{S}=2$ and $I \bar{S}=x y \bar{S}$ for some $x, y \in \mathfrak{n}$ whose images in $\bar{S}$ form a regular system of parameter of $\bar{S}$. Hence $\bar{R}=\bar{S} / x y \bar{S}$ is a hypersurface, in particular a complete intersection, of dimension one. Therefore $R$ is a complete intersection of dimension two by [7, Theorem 2.3.4(a)]. Since $S$ is a regular local ring of dimension three with regular system of parameter $x, y, z$, the ideal $I$ is generated by an $S$-sequence by [7, Theorem 2.3.3(c)]. Noting ht $I=\operatorname{dim} S-\operatorname{dim} R=1$, we see that $I$ is a principal ideal. Write $I=(l)$ for some $l \in I$. There is an element $f \in S$ such that $l=x y-z f$. Assume that $f \notin \mathfrak{n}$. Then $f$ is a unit of $S$, and we see that $z R \subseteq x y R$. Hence $\mathfrak{m}=(x, y) R$, and $\operatorname{edim} R=\operatorname{dim} R=2$. This implies that $R$ is regular, which is a contradiction. It follows that $f \in \mathfrak{n}$.

On the other hand, if $z \in \mathfrak{n}$ is an element whose image in $\mathfrak{m}$ is $R$-regular such that $\mathfrak{m} / z R$ is decomposable, then $z \notin \mathfrak{n}^{2}$. Indeed, assume $z \in \mathfrak{n}^{2}$. Then we have $I+(z) \subseteq \mathfrak{n}^{2}$. Since $R / z R=S / I+(z)$, it follows from Theorem 3.2.4 
that $\operatorname{dim} S=2$. Since $\operatorname{dim} R=2$, we have $I=0$, equivalently $R=S$. In particular $R$ is regular, which is a contradiction.

Thus, it suffices to show the existence of an $R$-regular element $w \in \mathfrak{m}$ such that $\mathfrak{m} /(w)$ is decomposable. Let $E$ denote the fundamental module of $R$. Proposition 2.4.4(4) says that we can write $E=M \oplus N$ for some non-zero $R$-modules $M$ and $N$. Hence the fundamental sequence of $R$ is as follows:

$$
0 \longrightarrow R \stackrel{\left(\begin{array}{l}
\sigma \\
\tau
\end{array}\right)}{\longrightarrow} M \oplus N \stackrel{(f, g)}{\longrightarrow} \mathfrak{m} \longrightarrow 0 .
$$

Take an $R$-regular element $w \in \mathfrak{m}-\mathfrak{m}^{2}$, and set $\overline{(-)}=(-) \otimes_{R} R /(w)$. If $\mathfrak{m} \bar{R}$ is decomposable, then our aim is attained. Hence let $\mathfrak{m} \bar{R}$ be indecomposable. The sequence (a) induces another exact sequence $0 \rightarrow \bar{R} \stackrel{\left(\frac{\bar{\sigma}}{\tau}\right)}{\longrightarrow}$ $\bar{M} \oplus \bar{N} \stackrel{(\bar{f}, \bar{g})}{\longrightarrow} \overline{\mathfrak{m}} \rightarrow 0$. (Here, the injectivity of the map $\left(\frac{\bar{\sigma}}{\bar{\tau}}\right)$ follows from the fact that $w$ is an m-regular element.) According to Lemma 3.3.4, the natural surjection $\pi: \overline{\mathfrak{m}} \rightarrow \mathfrak{m} \bar{R}$ is a split-epimorphism with kernel isomorphic to $k$. Hence there exists a split-monomorphism $\rho: \mathfrak{m} \bar{R} \rightarrow \overline{\mathfrak{m}}$ such that $\pi \rho=1$. Then note that the cokernel of $\rho$ is isomorphic to $k$. On the other hand (cf. Proposition 2.4.4), the homomorphism $(\bar{f}, \bar{g})$ is a $\mathcal{G}(\bar{R})$-precover of $\overline{\mathfrak{m}}$. Therefore there exists a homomorphism $\left(\begin{array}{c}\alpha \\ \beta\end{array}\right): \mathfrak{m} \bar{R} \rightarrow \bar{M} \oplus \bar{N}$ such that $\rho=(\bar{f}, \bar{g})\left(\begin{array}{l}\alpha \\ \beta\end{array}\right)=\bar{f} \alpha+\bar{g} \beta$. Set $e=\operatorname{edim} R, m=\nu_{R}(M)$, and $n=\nu_{R}(N)$.

Claim 1. We have either $(m, n)=(e-1,2)$ or $(m, n)=(2, e-1)$.

Proof. Since $\rho$ is a split-monomorphism, so is the homomorphism $\left(\begin{array}{l}\alpha \\ \beta\end{array}\right)$. There is a commutative diagram

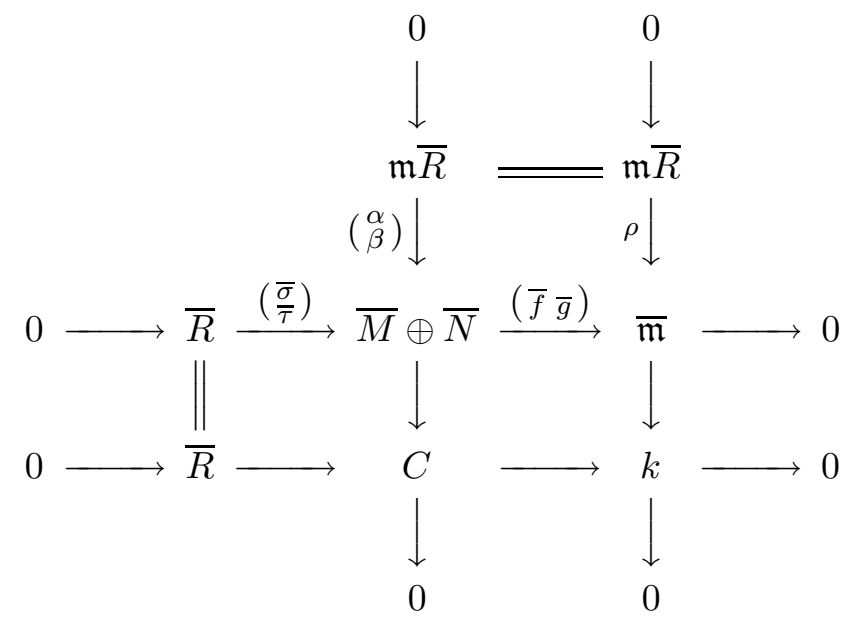


of $\bar{R}$-modules with exact rows and columns, and we have an isomorphism $\bar{M} \oplus \bar{N} \cong \mathfrak{m} \bar{R} \oplus C$. The indecomposability of $\mathfrak{m} \bar{R}$ and the Krull-Schmidt theorem yield that $\mathfrak{m} \bar{R}$ is isomorphic to a direct summand of either $\bar{M}$ or $\bar{N}$.

Let us consider the case where $\mathfrak{m} \bar{R}$ is isomorphic to a direct summand of $\bar{M}$. There is an $\bar{R}$-module $L$ such that $\bar{M} \cong \mathfrak{m} \bar{R} \oplus L$. The Krull-Schmidt theorem again yields an isomorphism

$$
C \cong \bar{N} \oplus L .
$$

Note that $\bar{N}$ and $L$ are isomorphic to direct summands of $\bar{E}$. Proposition 2.4.4 implies that the $\bar{R}$-module $\bar{E}$ belongs to $\mathcal{G}(\bar{R})$. The $\bar{R}$-modules $\bar{N}, L$ also belong to $\mathcal{G}(\bar{R})$, and so does $C$ by (b). Therefore the exact sequence

$$
0 \longrightarrow \bar{R} \longrightarrow C \longrightarrow k \longrightarrow 0
$$

in the above diagram does not split because depth $C=1>0$. On the other hand, noting that $\bar{R}$ is a Gorenstein local ring of dimension one, we have $\operatorname{Hom}_{\bar{R}}(k, \bar{R})=0$ and $\operatorname{Ext}_{\bar{R}}(k, \bar{R}) \cong k$. Dualizing the natural exact sequence $0 \rightarrow \mathfrak{m} \bar{R} \rightarrow \bar{R} \rightarrow k \rightarrow 0$, we have another exact sequence

$$
0 \longrightarrow \bar{R} \longrightarrow \operatorname{Hom}_{\bar{R}}(\mathfrak{m} \bar{R}, \bar{R}) \longrightarrow k \longrightarrow 0 \text {. }
$$

Note that the maximal ideal $\mathfrak{m} \bar{R}$ of $\bar{R}$ belongs to $\mathcal{G}(\bar{R})$, hence so does $\operatorname{Hom}_{\bar{R}}(\mathfrak{m} \bar{R}, \bar{R})$. Therefore the exact sequence (d) does not split because $\operatorname{depth} \operatorname{Hom}_{\bar{R}}(\mathfrak{m} \bar{R}, \bar{R})=1>0$.

Thus, we have obtained two non-split exact sequences (c) and (d) of $\bar{R}$-modules. Since $\operatorname{Ext} \frac{1}{R}(k, \bar{R}) \cong k$, we obtain an isomorphism

$$
C \cong \operatorname{Hom}_{\bar{R}}(\mathfrak{m} \bar{R}, \bar{R}) .
$$

The isomorphisms (b) and (e) give other isomorphisms $\mathfrak{m} \bar{R} \cong \operatorname{Hom}_{\bar{R}}\left(\operatorname{Hom}_{\bar{R}}\right.$ $(\mathfrak{m} \bar{R}, \bar{R}), \bar{R}) \cong \operatorname{Hom}_{\bar{R}}(\bar{N} \oplus L, \bar{R}) \cong \operatorname{Hom}_{\bar{R}}(\bar{N}, \bar{R}) \oplus \operatorname{Hom}_{\bar{R}}(L, \bar{R})$. Note that $\bar{N} \neq 0$ and $L$ are reflexive $\bar{R}$-modules, hence $\operatorname{Hom}_{\bar{R}}(\bar{N}, \bar{R}) \neq 0$. Since $\mathfrak{m} \bar{R}$ is indecomposable, we have $\operatorname{Hom}_{\bar{R}}(L, \bar{R})=0$, and hence $L=0$. Thus we get two isomorphisms $\bar{M} \cong \mathfrak{m} \bar{R}$ and $\bar{N} \cong \operatorname{Hom}_{\bar{R}}(\mathfrak{m} \bar{R}, \bar{R})$. Therefore $m=\nu_{\bar{R}}(\bar{M})=\operatorname{edim} \bar{R}=e-1$ because $w \notin \mathfrak{m}^{2}$, and $n=\nu_{\bar{R}}(\bar{N})=$ $\nu_{\bar{R}}\left(\operatorname{Hom}_{\bar{R}}(\mathfrak{m} \bar{R}, \bar{R})\right)$. Lemma 3.3.5 implies that $n \geq 2$. On the other hand, 
it follows from the fundamental sequence (a) that $m+n=\nu_{R}(M \oplus N) \leq$ $\nu_{R}(R)+\nu_{R}(\mathfrak{m})=1+e$. Hence we see that $n=2$.

In the case where $\mathfrak{m} \bar{R}$ is isomorphic to a direct summand of $\bar{N}$, a similar argument yields $m=2$ and $n=e-1$.

On the other hand, we have $1=\pi \rho=\pi \bar{f} \alpha+\pi \bar{g} \beta$ in $\operatorname{End}_{\bar{R}}(\mathfrak{m} \bar{R})$. Since $\mathfrak{m} \bar{R}$ is indecomposable, the endomorphism ring $\operatorname{End}_{\bar{R}}(\mathfrak{m} \bar{R})$ is a local ring (cf. [15, Proposition (1.18)]), and hence either $\pi \bar{f} \alpha$ or $\pi \bar{g} \beta$ is a unit of this ring, in other words, is an automorphism. Put $\mathfrak{a}=\operatorname{Im} f$ and $\mathfrak{b}=\operatorname{Im} g$.

Claim 2. If $\pi \bar{f} \alpha$ (resp. $\pi \bar{g} \beta)$ is an automorphism, then $\mathfrak{m}=\mathfrak{a}+(w)$ $($ resp. $\mathfrak{m}=\mathfrak{b}+(w))$ and grade $\mathfrak{a}>0$ (resp. grade $\mathfrak{b}>0)$.

Proof. Suppose that $\pi \bar{f} \alpha$ is an automorphism. Then $\pi \bar{f}$ is a splitepimorphism, and so in particular a surjection. Hence $\mathfrak{m} \bar{R}=\mathfrak{a} \bar{R}$, and therefore $\mathfrak{m}=\mathfrak{a}+(w)$. There exists an $\bar{R}$-regular element in $\mathfrak{m} \bar{R}=\mathfrak{a} \bar{R}$. We can choose an element $v \in \mathfrak{a}$ whose image in $\mathfrak{m} \bar{R}$ is $\bar{R}$-regular. Since $w, v$ is an $R$-regular sequence, so is the sequence $v, w$. Thus $v$ is an $R$-regular element. The proof of the other case is similar.

Claim 3. We have both grade $\mathfrak{a}>0$ and grade $\mathfrak{b}>0$.

Proof. It is enough to show the claim only in the case where $\pi \bar{f} \alpha$ is an automorphism. Then Claim 2 says that $\mathfrak{m}=\mathfrak{a}+(w)$ and grade $\mathfrak{a}>0$. Take an $R$-regular element $v \in \mathfrak{a}-\mathfrak{m}^{2}$. Applying the above argument to the element $v$ instead of $w$, we see that either of the following holds:

(i) $\mathfrak{m}=\mathfrak{a}+(v)$ and grade $\mathfrak{a}>0$;

(ii) $\mathfrak{m}=\mathfrak{b}+(v)$ and grade $\mathfrak{b}>0$.

However, if the statement (i) holds, then we have $\mathfrak{m}=\mathfrak{a}$, which means that the homomorphism $f: M \rightarrow \mathfrak{m}$ is surjective. Hence $m=\nu_{R}(M) \geq$ $\nu_{R}(\mathfrak{m})=e$. It follows from Claim 1 that $m=2$, and hence $e \leq 2$. But this can not happen because $R$ is a non-regular local ring of dimension two. Consequently the statement (ii) must hold, and we obtain grade $\mathfrak{b}>0$, as desired.

Put $x=\sigma(1)$ and $y=\tau(1)$. Then $f(x)+g(y)=(f, g)\left(\begin{array}{c}\sigma \\ \tau\end{array}\right)(1)=0$. Set $v=f(x)=-g(y) \in \mathfrak{a} \cap \mathfrak{b}$. Take an element $a \in \mathfrak{a} \cap \mathfrak{b}$. Then we have $a=f(p)=g(q)$ for some $p \in M$ and $q \in N$. Hence $\left(\begin{array}{c}p \\ -q\end{array}\right) \in \operatorname{Ker}(f, g)=$ 
$\operatorname{Im}\left(\begin{array}{c}\sigma \\ \tau\end{array}\right)$, and therefore $\left(\begin{array}{c}p \\ -q\end{array}\right)=b\left(\begin{array}{l}x \\ y\end{array}\right)$ for some $b \in R$. Thus $p=b x$, and we get $a=f(p)=f(b x)=b v \in(v)$. It follows that $\mathfrak{a} \cap \mathfrak{b}=(v)$. Since grade $(v)=$ $\operatorname{grade}(\mathfrak{a} \cap \mathfrak{b})=\inf \{\operatorname{grade} \mathfrak{a}$, grade $\mathfrak{b}\}>0$ by [7, Proposition 1.2.10(c)] and Claim 3, the element $v$ is an $R$-regular element.

Set $\overline{(-)}=(-) \otimes_{R} R /(v)$. Since $\mathfrak{a}+\mathfrak{b}=\mathfrak{m}$ and $\mathfrak{a} \cap \mathfrak{b}=(v)$, there is a natural exact sequence $\omega: 0 \rightarrow \bar{R} \rightarrow R / \mathfrak{a} \oplus R / \mathfrak{b} \rightarrow k \rightarrow 0$ of $\bar{R}$-modules. Suppose that this exact sequence splits. Then we have an isomorphism $R / \mathfrak{a} \oplus R / \mathfrak{b} \cong \bar{R} \oplus k$, and it is seen from the Krull-Schmidt theorem that $k$ is isomorphic to either $R / \mathfrak{a}$ or $R / \mathfrak{b}$. Hence we have either $\mathfrak{m}=\mathfrak{a}$ or $\mathfrak{m}=\mathfrak{b}$, and the same argument as the end of the proof of Claim 3 yields a contradiction. Thus the exact sequence $\omega$ does not split.

On the other hand, dualizing the natural exact sequence $0 \rightarrow \mathfrak{m} \bar{R} \rightarrow$ $\bar{R} \rightarrow k \rightarrow 0$, we have a non-split exact sequence $0 \rightarrow \bar{R} \rightarrow \operatorname{Hom}_{\bar{R}}(\mathfrak{m} \bar{R}, \bar{R}) \rightarrow$ $k \rightarrow 0$. Since $\operatorname{Ext} \frac{1}{R}(k, \bar{R}) \cong k$, we obtain an isomorphism $R / \mathfrak{a} \oplus R / \mathfrak{b} \cong$ $\operatorname{Hom}_{\bar{R}}(\mathfrak{m} \bar{R}, \bar{R})$, and $\operatorname{Hom}_{\bar{R}}(\mathfrak{m} \bar{R}, \bar{R})$ belongs to $\mathcal{G}(\bar{R})$. It follows that both $R / \mathfrak{a}$ and $R / \mathfrak{b}$ belong to $\mathcal{G}(\bar{R})$, hence they are reflexive over $\bar{R}$. Therefore the $\bar{R}$-dual modules $\operatorname{Hom}_{\bar{R}}(R / \mathfrak{a}, \bar{R})$ and $\operatorname{Hom}_{\bar{R}}(R / \mathfrak{b}, \bar{R})$ are non-zero, which proves that $\mathfrak{m} \bar{R}$ is decomposable. This completes the proof of our theorem.

Combining Theorem 3.3.1 with Theorem 3.3.2 gives birth to the following corollary. Compare it with Corollary 3.2.5.

Corollary 3.3.6. Let $(R, \mathfrak{m}, k)$ be a complete local ring. Suppose that $\mathfrak{m}$ is indecomposable. Then the following conditions are equivalent:

(1) $\Omega_{R}^{2} k$ has a non-zero proper direct summand of finite G-dimension;

(2) $R$ is Gorenstein, and $\Omega_{R}^{2} k$ is decomposable;

(3) There are a complete regular local ring $(S, \mathfrak{n})$ of dimension three, a regular system of parameters $x, y, z$ of $S$, and $f \in \mathfrak{n}$ such that $R \cong$ $S /(x y-z f)$.

Lastly, we recall a result of Yoshino and Kawamoto, which is related to Theorem 3.3.2. A homomorphic image of a convergent power series ring over a field $k$ is called an analytic ring over $k$. Any complete local ring containing a field is an analytic ring over its coefficient field, and it is known that any analytic local ring is Henselian; see [12, Chapter VII]. Yoshino and Kawamoto observed the decomposability of the fundamental module of an analytic normal domain. 
Theorem 3.3.7. (Yoshino-Kawamoto) Let $R$ be an analytic normal local domain of dimension two. Suppose that the residue class field of $R$ is algebraically closed and has characteristic zero. Then the following conditions are equivalent:

(1) The fundamental module of $R$ is decomposable;

(2) $R$ is an invariant subring of a regular local ring by a cyclic group. (In other words, $R$ is a cyclic quotient singularity.)

For the details of this theorem, see [17, Theorem (2.1)] or [15, Theorem (11.12)]. With the notation of the above theorem, suppose in addition that $R$ is a complete Gorenstein ring such that $\Omega_{R}^{2} k$ is decomposable. Then it is seen from Proposition 2.4.4(4) that $R$ satisfies the condition (1) in the above theorem. Hence the proof of the above theorem shows that $R$ is of finite Cohen-Macaulay type; see [17] or [15]. It follows from a theorem of Herzog [10] that $R$ is a hypersurface. Therefore the local ring $R$ is a rational double point of type $\left(A_{n}\right)$ for some $n \geq 1$ by [17, Proposition (4.1)], namely, $R \cong k[[X, Y, Z]] /\left(X Y-Z^{n+1}\right)$. Thus, the second condition of Theorem 3.3.2 holds.

Acknowledgments. The author would like to extend hearty thanks to Shiro Goto. He motivated the author to consider such a ring as in Proposition 3.1.2, which led the author to write this paper. The author also wishes to express his deep gratitude to Yuji Yoshino, who gave the author a lot of valuable comments. In particular, Claim 1 in the proof of Theorem 3.3.2 is due to him. The author would also like to thank an anonymous referee very much for useful comments and suggestions.

\section{REFERENCES}

[1] M. Auslander, Anneaux de Gorenstein, et torsion en algèbre commutative, Séminaire d'Algèbre Commutative dirigé par Pierre Samuel, 1966/67. Texte rédigé, d'après des exposés de Maurice Auslander, Marquerite Mangeney, Christian Peskine et Lucien Szpiro. École Normale Supérieure de Jeunes Filles, Secrétariat mathématique, Paris, 1967.

[2] M. Auslander, Minimal Cohen-Macaulay approximations, unpublished paper.

[3] M. Auslander and M. Bridger, Stable module theory, Memoirs of the American Mathematical Society, No. 94, American Mathematical Society, Providence, R.I., 1969.

[4] M. Auslander, S. Ding and Ø. Solberg, Liftings and weak liftings of modules, J. Algebra, 156 (1993), 273-317. 
[5] L. L. Avramov, Infinite free resolutions, Six lectures on commutative algebra (Bellaterra, 1996), Progr. Math., 166, Birkhauser, Basel, 1998, pp. 1-118.

[6] L. L. Avramov, Homological dimensions and related invariants of modules over local rings, Representations of Algebras, ICRA IX (Beijing, 2000), vol. I, Beijing Normal Univ. Press, 2002, pp. 1-39.

[7] W. Bruns and J. Herzog, Cohen-Macaulay rings, revised edition, Cambridge Studies in Advanced Mathematics, 39, Cambridge University Press, Cambridge, 1998.

[8] L. W. Christensen, Gorenstein dimensions, Lecture Notes in Mathematics, 1747, Springer-Verlag, Berlin, 2000.

[9] S. P. Dutta, Syzygies and homological conjectures, Commutative algebra (Berkeley, CA, 1987), Math. Sci. Res. Inst. Publ., 15, Springer, New York, 1989, pp. 139-156.

[10] J. Herzog, Ringe mit nur endlich vielen Isomorphieklassen von maximalen, unzerlegbaren Cohen-Macaulay-Moduln, Math. Ann., 233 (1978), no. 1, 21-34.

[11] A. Martsinkovsky, A remarkable property of the (co) syzygy modules of the residue field of a nonregular local ring, J. Pure Appl. Algebra, 110 (1996), no. 1, 9-13.

[12] M. Nagata, Local rings, Interscience Tracts in Pure and Applied Mathematics, No. 13, Interscience Publishers, John Wiley \& Sons, New York-London, 1962.

[13] R. Takahashi, On the category of modules of Gorenstein dimension zero II, J. Algebra, 278 (2004), no. 1, 402-410.

[14] J. Tate, Homology of Noetherian rings and local rings, Illinois J. Math., 1 (1957), $14-27$.

[15] Y. Yoshino, Cohen-Macaulay modules over Cohen-Macaulay rings, London Mathematical Society Lecture Note Series, 146, Cambridge University Press, Cambridge, 1990.

[16] Y. Yoshino, Cohen-Macaulay approximations (Japanese), Proceedings of the 4th Symposium on Representation Theory of Algebras, Izu, Japan, 1993, pp. 119-138.

[17] Y. Yoshino and T. Kawamoto, The fundamental module of a normal local domain of dimension 2, Trans. Amer. Math. Soc., 309 (1988), no. 1, 425-431.

Department of Mathematics

School of Science and Technology

Meiji University

Kawasaki 214-8571

Japan

takahasi@math.meiji.ac.jp

Current AdDress:

Department of Mathematical Sciences

Faculty of Science

Shinshu University

Matsumoto 390-8621

Japan

takahasi@math.shinshu-u.ac.jp 